\title{
Iridoschisis: case report and review of the literature
}

This article was published in the following Dove Press journal:

Clinical Ophthalmology

17 March 201।

Number of times this article has been viewed

\section{Eleni Gogaki}

Fani Tsolaki

Sotiria Tiganita

Christina Skatharoudi

Demetrios Balatsoukas

Ophthalmology Department, Hippokration General Hospital, Thessaloniki, Greece
Correspondence: Eleni Gogaki Olympou I06B, 55236, Panorama,

Thessaloniki, Greece

Tel +3069446888I5

Email egogaki@hotmail.com
Abstract: Iridoschisis is a rare condition that consists of the separation of the anterior mesodermic layer of the iris. In more than two-thirds of cases it is associated with glaucoma. We report the case of an 80-year-old patient who had bilateral iridoschisis and presented with acute angle-closure glaucoma. The patient was free from a history of ocular trauma or of heritable ocular disease. Chronic open-angle glaucoma, as well as intermittent angle-closure glaucoma, should be excluded in all patients with iridoschisis, and regular follow-up should be established upon diagnosis of the entity.

Keywords: iridoschisis, angle-closure glaucoma, iris degeneration

\section{Introduction}

Iridoschisis is a rare condition in which the iris stroma is cleaved in two portions and is defined as a separation of the anterior iris stroma from the posterior stroma and muscle layers. The anterior layer splits into strands, and the free ends float freely in the anterior chamber. ${ }^{1}$

The condition was first described in 1922 by Schmitt when he presented a case with detachment of the anterior iris layer. ${ }^{2}$ The term "iridoschisis" was first introduced by Loewenstein and Foster in 1945, when they described a case of end-stage glaucoma in an old woman who had an inferior localized cleavage of the iris into two layers, with the anterior fibrils of the iris waving in the anterior chamber. ${ }^{3}$

\section{Case presentation}

An 80-year-old woman was referred to the emergency unit of our ophthalmology department where she presented with a history of acute and persistent pain in the left eye and temple, accompanied by nausea and vomiting during the previous 24 hours.

The clinical examination showed that the best-corrected visual acuity of the affected eye was finger count (at $1.5 \mathrm{~m}$ ), and the intraocular pressure at the time of admission was $54 \mathrm{mmHg}$. The conjunctiva was intensely red, with corneal injection, and mild edema was seen in the corneal stroma. The anterior chamber was remarkably shallow and showed loose ends of degenerated anterior stromal leaf fibrils floating freely in the aqueous. The iris showed typical features of iridoschisis, ie, stromal separation with cystic iris elevation in the inferior half and forward bowing of the iris (Figure 1). The pupil was semidilated and nonreacting to light. However, the indirect pupillary reflex was produced in the right eye. The lens showed mature cataract which hindered visualization of the fundus and evaluation of any optic disc alterations. Slit-lamp 


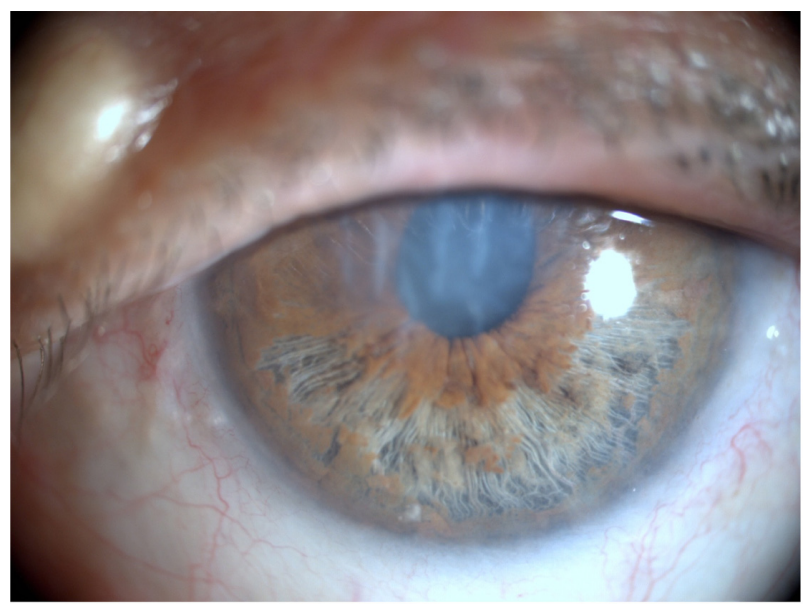

Figure I The anterior chamber is remarkably shallow and presents loose ends of degenerated anterior stromal leaf fibrils floating freely in the aqueous. The iris shows the typical features of iridoschisis, that is, stromal separation with cystic iris elevation in the inferior half and forward bowing of the iris (left eye).

examination of the fellow eye revealed similar features, with a shallow anterior chamber and iridoschisis concerning the inferior half of the tissue (Figure 2). The patient was free from a history of ocular trauma or of heritable ocular disease. She mentioned that during the previous years she suffered from frequent episodes of painful "conjunctivitis" occurring at any time of the year with no mucopurulent secretion or pruritus, affecting either eye during an attack. She was on antihypertensive treatment. The patient was diagnosed as having acute angle-closure glaucoma, and was receiving acetazolamide $250 \mathrm{mg}$ three times daily, $\operatorname{Cosopt}^{\circledR}$ twice daily, isoptocarpine every two hours, intravenous mannitol $(1 \mathrm{~g} / \mathrm{kg}$ body weight stat), and oral pain-relieving drugs.

On the third day after admission, the intraocular pressure of the affected eye was regulated at $14 \mathrm{mmHg}$ and the

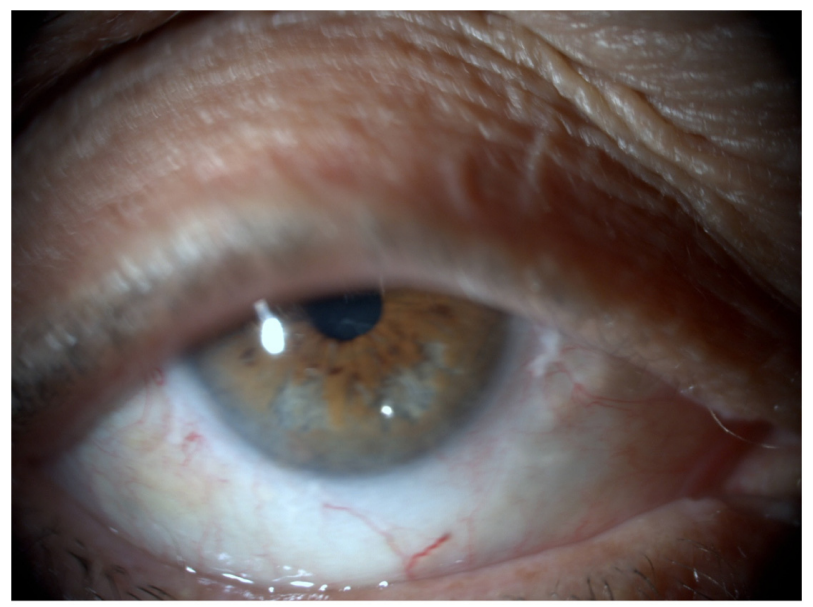

Figure 2 Right eye, reveals similar features with a shallow anterior chamber and iridoschisis concerning the inferior half of the tissue. administration of acetazolamide and Cosopt was gradually discontinued. Slit-lamp biomicroscopy showed that the cornea was no longer edematous and gonioscopy was therefore possible. We observed that there was a closed angle (Shaffer grade $0-1$ ) for $270^{\circ}$ and scattered peripheral anterior synechiae in the superior quadrant of both eyes. Due to advanced cataract, it was not feasible to perform visual field examination.

Laboratory investigations showed a normal full blood count, prolonged prothrombin time, and increased INR. There were slightly increased values for urea, uric acid, and C-reactive protein $(6.03 \mathrm{mg} / \mathrm{L})$. The erythrocyte sedimentation rate was $58 \mathrm{~mm}$ for the first hour. Observing that the lesion was in the iris stroma, we wanted to exclude whether this could be attributed to a connective tissue disease, so did an immunoserologic profile examination. The values for rheumatoid factor, antinuclear antibodies, antimitochondrial antibodies, antineutrophil cytoplasmic antibodies, and anticardiolipin antibodies were all within normal range. These immunoserologic assays are part of the standard procedure used in our screening protocol for exclusion of autoimmune connective tissue disease.

Intraocular pressure measurements were taken three times daily. On the fifth day after her admission, when intraocular pressure measurements were within normal limits and showed no fluctuation, and the cornea and anterior chamber presented no inflammatory activity, the patient was ready to leave the clinic with a regimen for topical antiglaucoma treatment (isoptocarpine twice daily). The patient was extensively informed about the association of iridoschisis and the appearance of glaucoma, and she was advised to have frequent ophthalmologic examination, monitoring of intraocular pressure, and assessment for any progression of glaucomatous changes in both eyes.

\section{Discussion}

Iridoschisis is characterized by separation of the anterior and posterior stroma of the iris. The anterior layer is usually divided into a loose mixture of large number of pigmented and white atrophic strands running in all directions, but mostly from the periphery to the collarette. ${ }^{4}$ Larger free sections may contain blood vessels. It has been reported by fluoroiridography that there is normal perfusion of blood vessels, from the inner pupillary margin to the outer iris of the affected sectors. ${ }^{5}$ Histopathologic study of the iris showed tissue fibrosis and atrophy and evidence of several small clefts in the substance. The vessels did not look abnormal, although the endothelial lining of some of them might be swollen. ${ }^{6}$ Occasionally, separation at the posterior stroma 
might occur with irregular pigmentation of the pigment layers. $^{7}$

Electron transmission microscopy of the iris showed significant thinning of the stroma and a diminished number of collagen fibrils in the affected area, whereas the appearance of the stromal blood vessels and nerves was normal. ${ }^{7}$ Corneal changes were uncommon and, if present, degenerated corneal endothelial cells were localized over the area of iridoschisis, where the strands of degenerated iris may come into contact with the corneal endothelium. ${ }^{7}$

The causative agent of iridoschisis is not yet identified. Lowenstein and Foster suggested that cleavage of the iris in this particular plane may have an anatomic basis and that there has to be an atrophic effect of lytic substances in the aqueous, originating from a glaucomatous condition, or that the condition could be attributed to senile changes. ${ }^{3}$

Albers and Klein also ascribe the changes in the iris to the aging process and they propose that, as the sclerosis of blood vessels increases in the anterior iris stroma, a shearing action tears the tissue between the anterior and posterior sections during constriction and dilatation of the iris. ${ }^{6}$ Bojer suggests that iridoschisis is a form of essential iris atrophy in the elderly. ${ }^{8}$ Progressive iris atrophy begins in the third decade of life; iridoschisis, on the other hand, is seen mainly after the seventh decade of life. Other authors have shown that both the vasculature and its perfusion are normal in iridoschisis, in contrast with the vasculature of essential iris atrophy. ${ }^{5,7}$ Loewenstein et al have proposed that iridoschisis might be the result of trauma, and they assumed that a posttraumatic peak in intraocular pressure, which shears along the dilator fibers, could lead to splitting of the anterior and posterior iris stroma. ${ }^{9}$

An important aspect of iridoschisis is the frequent association with glaucoma. About two-thirds of reported cases of iridoschisis were seen in association with glaucoma.,10-12 Salmon and Murray studied a number of patients with iridoschisis and coexistent primary angle-closure glaucoma to determine the clinical features of the condition and to examine the relationship of iridoschisis to primary angle-closure glaucoma. Their study suggests that iridoschisis is an unusual manifestation of iris stromal atrophy, and results from intermittent or acute elevation of intraocular pressure. They proposed that primary angleclosure glaucoma should be excluded in patients who present with iridoschisis..$^{13}$ Romano et al report that iridoschisis precedes the angle-closure episode. ${ }^{11}$

Shima et al presented a case of iridoschisis and plateau iris configuration evidenced by ultrasound biomicroscopy. ${ }^{14}$ The patient had narrow angles and scattered peripheral anterior synechiae in both eyes. Ultrasound biomicroscopy showed anteriorly positioned ciliary body, slit-like narrowing of the iridocorneal angle, and a flat iris in all four quadrants in both eyes. This case points to the idea that iridoschisis might be a part of a general anterior anatomic malformation of ocular structures, together with plateau iris configuration.

Iridoschisis might also possess a familial character, because there is a report of a family with iridoschisis, narrow anterior chamber angle, and presenile cataract. The author proposes that family members should be screened for iridoschisis, as well as for associated ocular abnormalities. ${ }^{15}$

Iridoschisis is reported to be associated with lens subluxation, either anterior subluxation pushing the iris forward, shallowing the anterior chamber and causing angle-closure glaucoma, ${ }^{16}$ or posterior subluxation into the vitreous cavity. ${ }^{17} \mathrm{~A}$ subluxated lens rubbing against the iris may be a mechanical precipitating factor in the development of iridoschisis. ${ }^{16}$

There have been a number of cases reported to have iridoschisis and interstitial keratitis due to syphilis. ${ }^{18-20}$ Patients with congenital syphilis, apart from showing systemic features of congenital disease, presented with interstitial keratitis, ghost vessels, and iridoschisis involving predominantly the inferior half of the iris, and some of the patients had open-angle glaucoma. Corectopia and ectropion uvea were absent. ${ }^{21}$ No peripheral anterior synechiae or fibrovascular membranes were seen in patients with open-angle glaucoma. Neither interstitial keratitis nor iridoschisis had a suppurative component, and both were presumably immunologic in their pathogenesis. $^{18}$

In the differential diagnosis of iridoschisis are the two other main iris stromal anomalies, namely, the iridocorneal endothelial syndrome and neurocristopathy of the iris and cornea (Axenfeld-Rieger syndrome). Diagnosis is made on clinical appearance, age of onset, laterality, and whether the condition is congenital, progressive, or with coexistent glaucoma. ${ }^{5,22,23}$ The age of onset for iridocorneal endothelial syndrome is the third or fourth decade and is unilateral and progressive, while iridoschisis appears later, usually in the sixth to seventh decade, and is generally bilateral, symmetrical, and progressive. On the other hand, AxenfeldRieger syndrome is present at birth, has a congenital character, and is bilateral, asymmetrical, and nonprogressive. In iridoschisis, the iris displays stromal splitting without any holes, is sectorial, is mainly inferiorly located, with anterior stromal atrophy and strands floating in the anterior chamber. The pupil is round and reactive to light and accommodation, and there are usually no corneal changes, unless anterior iris 
strands reach to the endothelium. In iridocorneal endothelial syndrome, stromal atrophy of the iris is observed, with or without holes, and peripheral anterior synechiae extending to Schwalbe's line are present. The pupil may be ectopic, polycoria may even be observed, and the cornea manifests abnormal endothelium with pleomorphism and cell loss. In Axenfeld-Rieger syndrome, there is mild stromal thinning of the iris, atrophy, and hole formation, and peripheral anterior synechiae are observed on the anteriorly displaced Schwalbe's line. The pupil may be irregular, and the cornea presents with endothelial changes related to peripheral anterior synechiae. ${ }^{1,22-25}$ According to the aforementioned clinical entities, our patient had the differential characteristics of iridoschisis, although the exclusion of a diagnosis of iridocorneal endothelial syndrome or Axenfeld-Rieger syndrome can be sometimes difficult.

\section{Conclusion}

An 80-year-old patient presented with an episode of acute angle-closure glaucoma and iridoschisis. The finding of iridoschisis is a warning for the ophthalmologist to exclude any association with glaucoma, because two-thirds of patients with iridoschisis have glaucoma. It is also possible that a patient may develop glaucoma in the future, so standard glaucoma tests (including visual acuity, visual field assessment, tonometry, gonioscopy, biomicroscopy, and assessment of the optic nerve head) and regular follow-up are required.

\section{Disclosure}

The authors report no conflicts of interest in this work.

\section{References}

1. Schoneveld PG, Pesudovs K. Iridoschisis. Clin Exp Optom. 1999;82: 29-33.

2. Schmitt A. Ablosung des vorderen irisblattes. Augenh Klin Mbl. 1922; 68:214-215.
3. Loewenstein A, Foster J. Iridoschisis with multiple rupture of stromal threads. Br J Ophthalmol. 1945;29:277-282.

4. Nirankari MS, Singh D, Parkash OM. Iridoschisis. J All India Ophthalmol Soc. 1964;12:29-31.

5. Carnevalini A, Menchini U, Bandello F, et al. Fluoroiridographic aspects of iridoschisis. J Fr Ophtalmol. 1988;11:329-332. French.

6. Albers EC, Klien BA. Iridoschisis: A clinical and histopathologic study. Am J Ophthalmol. 1958;46:794-802.

7. Rodrigues MC, Spaeth GL, Krachmer JH, et al. Iridoschisis associated with glaucoma and bullous keratopathy. Am J Ophthalmol. 1983;95: $73-81$.

8. Bøjer J. Iridoschisis: Essential iris atrophy. Acta Ophthalmol. 1953;31: 253-264.

9. Loewenstein A, Foster J, Sledge SK. A further case of iridoschisis. $\mathrm{Br} J$ Ophthalmol. 1948;32:129-134.

10. Mills PV. Iridoschisis. Br J Ophthalmol. 1967;51:158-164.

11. Romano A, Treister G, Barishak R, et al. Iridoschisis and angle-closure glaucoma. Ophthalmologica. 1972;164:199-207.

12. McCulloch C. Iridoschisis as a cause of glaucoma. Am J Ophthalmol. 1950;33:1398-1400.

13. Salmon JF, Murray AD. The association of iridoschisis and primary angle-closure glaucoma. Eye (Lond). 1992;6:267-272.

14. Shima C, Otori Y, Miki A, et al. A case of iridoschisis associated with plateau iris configuration. Jpn J Ophthalmology. 2007;51:390-391.

15. Mansour AM. A family with iridoschisis, narrow anterior chamber angle, and presenile cataract. Ophthalmic Paediatr Genet. 1986;7:145-149.

16. Agrawal S, Agrawal J, Agrawal TP. Iridoschisis associated with lens subluxation. J Cataract Refract Surg. 2001;27:2044-2046.

17. Mutoh T, Matsumoto Y, Chikuda M. A case of iridoschisis associated with lens displacement into the vitreous cavity. Clin Ophthalmol. 2010;4:487-491.

18. Foss AJ, Hykin PG, Benjamin L. Interstitial keratitis and iridoschisis in congenital syphilis. J Clin Neuroophthalmol. 1992;12:167-170.

19. Salvador F, Linares F, Merita I, et al. Unilateral iridoschisis associated with syphilitic interstitial keratitis and glaucoma. Ann Ophthalmol. 1993;25:328-329.

20. Pearson PA, Amrien JM, Baldwin LB, et al. Iridoschisis associated with syphilitic interstitial keratitis. Am J Ophthalmol. 1989;107:88-90.

21. Duke-Elder S. Disease of the uveal tract. In: Duke-Elder S, editor. System of Ophthalmology. St. Louis: CV Mosby; 1966:694-698.

22. Shields MB. Progressive essential iris atrophy, Chandler's syndrome and iris nevus (Cogan-Reese) syndrome. A spectrum of disease. Surv Ophthalmol. 1979;24:3-20.

23. Shields MB, Buckley E, Klintworth GK, et al. Axenfeld-Rieger syndrome. A spectrum of developmental disorders. Surv Ophthalmol. 1985;29:387-409.

24. Kanski JJ. Glaucoma. In: Kanski JJ, editor. Clinical Ophthalmology. 6th ed. Oxford, UK: Butterworth Heinemann; 2007:412-415.

25. Scheie HG, Yannof M. Iris nevus (Cogan-Reese) syndrome: A cause of unilateral glaucoma. Arch Ophthalmol. 1975;93:963-970.
Clinical Ophthalmology

\section{Publish your work in this journal}

Clinical Ophthalmology is an international, peer-reviewed journal covering all subspecialties within ophthalmology. Key topics include: Optometry; Visual science; Pharmacology and drug therapy in eye diseases; Basic Sciences; Primary and Secondary eye care; Patient Safety and Quality of Care Improvements. This journal is indexed on Submit your manuscript here: http://www.dovepress.com/clinical-ophthalmology-journal

\section{Dovepress}

PubMed Central and CAS, and is the official journal of The Society of Clinical Ophthalmology (SCO). The manuscript management system is completely online and includes a very quick and fair peer-review system, which is all easy to use. Visit http://www.dovepress.com/ testimonials.php to read real quotes from published authors. 